\title{
Normalization of Images from Geostationary Earth Remote Sensing Systems
}

\author{
N. Egoshkin, V. Eremeev
}

Ryazan State Radio Engineering University, Gagarin Str., 59/1, Ryazan, Russia, 390005

Keywords: geometrical correction, image normalization, geodetic connection, geostationary satellites, geostationary observation.

\begin{abstract}
This paper considers models and algorithms of normalization of images obtained from geostationary remote sensing systems. And the paper suggests and researches algorithms of geometrical distortion correction and transformation of images into the standard Normalized Geostationary Projection. The algorithm of geodetic connection adjustment with usage of Earth contour points in the image was developed. Practical testing of algorithms in the images obtained from the Russian spacecraft "Elektro-L" was executed.
\end{abstract}

\section{Introduction}

Geostationary satellites are oriented to periodic (every 5-30 minutes) Earth image acquisition against the surrounding space background. Geostationary satellites are practically fixed relative to the Earth therefore the imagery system should have a two-axis scene scanning mechanism. This mechanism can be implemented in different ways. So the spacecraft GOMS revolves on its own axis [1]. The imagery is executed using linear arrays of charge-coupled device (CCD) detectors and a scanning mirror [2] in the modern spacecraft "Elektro-L".

Current requirements to image details (spatial resolution $\sim 1 \mathrm{~km}$ ) can be achieved only by means of push broom scanners (sensors) which form images by separate parts. Parts of the image can be formed simultaneously by several space-diversity linear arrays or by one linear array consecutively. The images formed by a sensor are geometrically distorted because of the scanning mechanism features. Besides different parts of the image have different distortions. Also errors of geodesic coordinate measurements arise because of the attitude measurement inaccuracy and satellite orientation.

The task of distortion correction and formation of a single normalized image from a set of parts in the normalized geostationary projection accepted by the international practice [3] arises.

\section{Algorithms of the image normalization}

\subsection{Models and algorithms of the geometrical correction}

2.1.1. Geometrical correction consists in formation of a new image with required geometrical characteristics from the original image and its representation in the normalized geostationary projection. The basis of this geometrical correction is mathematical models of imagery.

Let us consider an arbitrary linear array of CCD detectors. Then let's introduce the instrument coordinate system (ICS) so that its center coincides with a focus of $O$ lens and axis $Z$ - with an optical axis (Fig.1). 


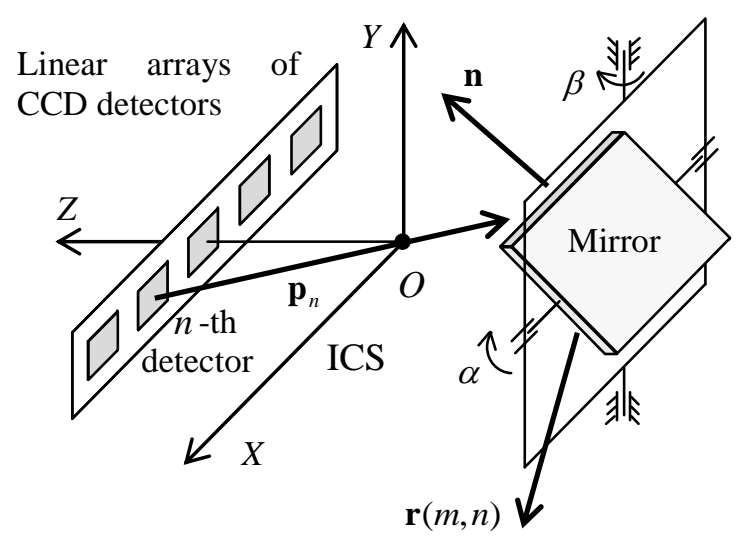

Figure 1. Imagery geometry of a sensor

Then a directing vector of an aiming beam that is incident on a mirror for $n$-CCD detector is determined as

$$
\mathbf{p}_{n}=-\left(x_{0}+n \Delta_{x}, y_{0}+n \Delta_{y}, f\right)^{\mathrm{T}},
$$

where $f-$ a focal length of lens, $x_{0}, y_{0}$ - coordinates of a central element of the linear array of CCD detectors, $\left(\Delta_{x}, \Delta_{y}\right)$ - a vector directed along the linear array of CCD detectors in the focal plane.

The directing vector $\mathbf{r}$ of the aiming beam reflected from the mirror has the form

$$
\mathbf{r}=\mathbf{p}-2(\mathbf{p}, \mathbf{n}) \mathbf{n},
$$

where $\mathbf{n}=(\cos \alpha,-\sin \beta \sin \alpha, \cos \beta \sin \alpha)^{\mathrm{T}}-$ a unit normal vector to the scanning mirror plane in the instrument coordinate system, this vector is determined by angles $\alpha, \beta$. These angles $\alpha, \beta$ are functions of a line number $m$ which either are specified under the development of a scanning drive or are measured directly for each line by special angle transmitters.

Then let's find coordinates of an aiming beam in the Greenwich coordinate system (GCS):

$$
\mathbf{r}_{g}=\mathbf{M}_{g}\left(t_{m}\right) \mathbf{M}_{u} \mathbf{r}, \quad t_{m}=t_{0}+\Delta t m,
$$

where $\mathbf{M}_{u}$ - a matrix of sensor setting angles; $\mathbf{M}_{g}(t)$ - a $t$ time-dependent matrix of the spacecraft orientation relative to the Greenwich coordinate system and this matrix is measured by navigation aids; $t_{0}, t_{m}$ - time of the first and $m$ line formation correspondingly; $\Delta t-$ a period of the detector linear array sampling.

Let's solve a task of intersection of the line specified parametrically $\mathbf{P}(l)=\mathbf{R}+l \mathbf{r}_{g}$ with an Earth ellipsoid

$$
X^{2} / a_{e}^{2}+Y^{2} / a_{e}^{2}+Z^{2} / b_{e}^{2}=1
$$

where $\mathbf{R}=\left(X_{0}, Y_{0}, Z_{0}\right)-$ a vector of the satellite position at the time $t_{m}$ according to the navigation aid data, $a_{e}$ and $b_{e}$ - equatorial and polar radiuses of the Earth ellipsoid. As a result coordinates of the Earth surface point $\mathbf{P}_{e}=\left(X_{e}, Y_{e}, Z_{e}\right)^{\mathrm{T}}$ are found.

Then the geodesic coordinates $(\lambda, \varphi)$ are determined for a point with Greenwich coordinates $\left(X_{e}, Y_{e}, Z_{e}\right)^{\mathrm{T}}$ :

$$
\lambda=\operatorname{acrtg}\left(Y_{e} / X_{e}\right), \varphi=\operatorname{acrtg}\left(Z_{e} \frac{a_{e}^{2}}{b_{e}^{2}} / \sqrt{X_{e}^{2}+Y_{e}^{2}}\right) .
$$

Finally coordinates $(x, y)$ of the normalized image point corresponding to $(\lambda, \varphi)$ are found according to the expressions describing the normalized geostationary projection [3]. 
2.1.2. Correlations $(1)-(5)$ describe the relation between coordinates $(x, y)$ of the normalized image $D(x, y)$ and coordinates of pixels $(m, n)$ in the original image. Let's designate mathematical correlations obtained in eq. (1)-(5) between $(x, y)$ and $(m, n)$ as certain functions

$$
x=F(m, n), \quad y=G(m, n) .
$$

Then formation of the normalized image $D(x, y)$ comes to the transformation of original images in the plane $x, y$. This action can be executed under the converse law [4]. Besides the numerical inversion [5] is used to find functions which are reverse to $F, G$. The inversion algorithm and algorithm of geometrical transformation describes in [5] are universal. However they do not take into account the structure of images formed as separate parts.

The functions $F, G$ do not have single-valued inverse functions because of imagery peculiarities, i.e. some couples $\left(m_{i}, n_{i}\right)$ can correspond to $(x, y)$. In order to provide high quality of the correction, it is necessary to accumulate information from several parts of the image by the following way:

$$
D(x, y)=\sum_{i} w_{i} B\left(m_{i}, n_{i}\right) / \sum_{i} w_{i}, \quad w_{i}=1-2\left|n_{i} / N\right|,
$$

where $N-$ a number of photo receiving elements. Weights $w_{i}$ in eq. (7) are specified so that they are decreasing from 1 to null as a pixel number $n_{i}$ is approaching to boundaries of the linear arrays of CCD detectors. This simple solution ensures a high-quality registration of neighbor partially overlapped images as far as variations in light intensity on the overlap region turn out to be smooth. If some parts are significantly overlapped, eq. (7) ensures an accumulation of information and increase of radiometric quality.

The calculation of eq. (7) can be carried out by two methods. In the first case each part of the original image is geometrically transformed separately. At the same time sums in the right and left parts (7) are accumulated in special buffers for each pixel. After this transformation the calculation of brightness for $D(x, y)$ is carried out by division for each pixel. Such approach requires additional memory for storage and accumulation of weights. In the second case the numerical inversion of $F$, $G$ is carried out so that the whole set of inverse coordinates $\left(m_{i}, n_{i}\right)$ can be determined for each pixel $(x, y)$. Such approach requires less memory but more computational efforts.

\section{Geodetic Connection of Images by Earth Contour Points}

Measurement uncertainty of the spacecraft position and orientation leads to inaccuracy regarding to the geodetic connection of objects in a normalized image. For example, the spacecraft "Elekto-L" errors concerning knowledge of orientation lead to geodetic connection errors up to $10-15 \mathrm{~km}$. The task of the error correction and high-precise geodetic connection arises.

There is a unique possibility to define more precisely the geodetic connection of some images obtained from the geostationary Earth remote sensing systems. These images contain the Earth reflecting against the surrounding space background. Ideally the boundary "earth-space" (the Earth outline) should be reflected in the image in a strongly defined position and have the ellipse form with known dimensions and semiaxis orientation. Such ideal Earth outline position can be strictly described mathematically and used as a standard for comparison with the real observable Earth outline.

Papers [2], [6-7] consider various issues of the geodetic connection adjustment of images obtained from geostationary satellites. However corresponding technologies are not applicable for the spacecraft "Elektro-L" because of low radiometric quality of original images.

The correlation extreme algorithm is suggested to be used for maximally stable search of the Earth counter points with usage of binary masks and preliminary normalization of images.

At first the geometrical transformation of original images is executed into an "additional" plane where counter points of the Earth form an ellipse. This important operation allows eliminating differences of geometrical distortions in various parts of the image. The quality of analyzable images and accuracy of geodetic connection increase correspondingly. Besides in the "additional" 
plane counter points of the Earth form a simple figure with known geometrical features and this figure can be used as a standard.

Let us write down the condition for the sensor aiming beam passing tangentially to surface of the Earth ellipsoid (4):

$$
\begin{aligned}
(\mathbf{N}, \mathbf{L})=0, \mathbf{L}=\left(X-X_{0}, Y-Y_{0}, Z-Z_{0}\right)^{\mathrm{T}}, \\
\mathbf{N}=\left(X / a_{e}^{2}, Y / a_{e}^{2}, Z / b_{e}^{2}\right)^{\mathrm{T}},
\end{aligned}
$$

where $\mathbf{L}$ - a directing vector of the aiming beam, $\mathbf{N}-$ a normal vector to the ellipsoid (4), $(X, Y, Z)$ - coordinates of the Earth point. After transformations (8) the following is received

$$
X X_{0} / a_{e}^{2}+Y Y_{0} / a_{e}^{2}+Z Z_{0} / b_{e}^{2}-1=0 \text {. }
$$

Equation (9) determines a plane with a determining vector $\mathbf{S}=\left(X_{0}, Y_{0}, Z_{0} a_{e}^{2} / b_{e}^{2}\right) /$ $\sqrt{X_{0}^{2}+Y_{0}^{2}+Z_{0}^{2} a_{e}^{4} / b_{e}^{4}}$. Since an ellipsoid and any other plane are crossed over the ellipse then the Earth counter points are located at the ellipse in plane (9).

Let us consider an additional plane passing through the point $(0,0,0)$ in parallel to plane (9):

$$
X X_{0}+Y Y_{0}+Z Z_{0} a_{e}^{2} / b_{e}^{2}=0 .
$$

Then let us transfer the ellipse from plane (9) in plane (10) by means of projection from the center $\left(X_{0}, Y_{0}, Z_{0}\right)$. Analyzing (8) - (10), it can be shown that the ellipse from plane (11) is also an ellipse with the center $(0,0,0)$ and semiaxes

$$
\begin{aligned}
a=a_{e} \sqrt{\frac{X_{0}^{2} b_{e}^{2}+Y_{0}^{2} b_{e}^{2}+Z_{0}^{2} a_{e}^{2}}{X_{0}^{2} b_{e}^{2}+Y_{0}^{2} b_{e}^{2}+Z_{0}^{2} a_{e}^{2}-a_{e}^{2} b_{e}^{2}}}, \\
b=\sqrt{\frac{X_{0}^{2} b_{e}^{4}+Y_{0}^{2} b_{e}^{4}+Z_{0}^{2} a_{e}^{4}}{X_{0}^{2} b_{e}^{2}+Y_{0}^{2} b_{e}^{2}+Z_{0}^{2} a_{e}^{2}-a_{e}^{2} b^{2}}} .
\end{aligned}
$$

Taking into account the symmetry it can be shown that the major semiaxis of this ellipse is directed along the vector $\mathbf{A}=\left(-Y_{0}, X_{0}, 0\right) / \sqrt{X_{0}^{2}+Y_{0}^{2}}$, but the minor semiaxis is perpendicular and is directed along the vector $\mathbf{B}=\mathbf{S} \times \mathbf{A}$.

Let us introduce the coordinate system $X^{\prime}, Y^{\prime}, Z^{\prime}$ so that its axes are directed along vectors $\mathbf{A}, \mathbf{B}, \mathbf{S}$ correspondingly and its center is located in plane (11) in the ellipse center. In this system the ellipse is described as

$$
\left(X^{\prime}\right)^{2} / a^{2}+\left(Y^{\prime}\right)^{2} / b^{2}=1 .
$$

So in the ideal case (in the absence of errors under the determination of the satellite position and orientation) counter points of the Earth in the coordinate system $X^{\prime}, Y^{\prime}$ form an ellipse (12) with known parameters (11). The geodetic connection parameters are estimated on the basis of analysis of deviations from the ellipse (11) of a real geometric figure of the Earth counter.

Let us determine functions of the coordinate compliance between $(m, n)$ and $\left(X^{\prime}, Y^{\prime}\right)$ by the following way. Firstly vector $\mathbf{r}_{g}$ is found for the point $(m, n)$ according to (1)-(3). Then vectors $\mathbf{r}_{g}$ and $\mathbf{R}$ are recalculated into the coordinate system $X^{\prime}, Y^{\prime}, Z^{\prime}$ :

Then slim axes $\left(X^{\prime}, Y^{\prime}\right)$ are determined as

$$
\mathbf{r}^{\prime}=\mathbf{M}^{\prime} \mathbf{r}_{g}, \quad \mathbf{R}^{\prime}=\mathbf{M}^{\prime} \mathbf{R}, \quad \mathbf{M}=\left[\begin{array}{ccc}
A_{X} & A_{Y} & A_{Z} \\
B_{X} & B_{Y} & B_{Z} \\
S_{X} & S_{Y} & S_{Z}
\end{array}\right] .
$$

$$
X^{\prime}=R_{X}^{\prime}-R_{Z}^{\prime} r_{X}^{\prime} / r_{Z}^{\prime}, \quad Y^{\prime}=R_{X}^{\prime}-R_{Z}^{\prime} r_{Y}^{\prime} / r_{Z}^{\prime} .
$$

In practice the image in the "additional" plane is formed using (1)-(3), (13), (14). Then it is necessary to determine a set of the Earth counter point coordinates in the image. Thereto the standard ellipse (12) is divided into $K$ sectors. The standard fragments $E_{i}, i=1,2, \ldots, K$ are formed for 
each sector where -1 and +1 correspond to the space and Earth parts adjacent to the ellipse boundaries (12):

where $\Theta(\xi, \zeta)=\left\{(x, y): \xi^{2}<x^{2}+y^{2} a^{2} b^{2} \leq \zeta^{2}\right\}$,

$$
E_{i}(x, y)=\left\{\begin{array}{l}
+1, \quad(x, y) \in \Theta(a-\Delta, a) \cap S_{i}, \\
-1, \quad(x, y) \in \Theta(a, a+\Delta) \cap S_{i}, \\
0, \text { otherwise }
\end{array}\right.
$$

$$
S_{i}=\left\{(x, y): 2 \pi i / K<\pi+\arctan \frac{y}{x}<2 \pi(i+1) / K\right\} .
$$

Then the correlation extreme search of standard fragments $E_{i}$ is carried out in the image. The standard coordinates of the search zone centres have the form $\mathbf{P}_{i}=$ $(-a \cos 2 \pi(i+0.5) / K,-b \sin 2 \pi(i+0.5) / K), \quad i=1,2, \ldots, K$. Since the standard fragments are binary then calculation of the correlation coefficient (CC) requires only addition and subtraction operations. Besides changes of $\mathrm{CC}$ under the $E_{i}$ offset in the search zone are connected only with boundary points of the null and unit region at $E_{i}$ that also reduces the number of calculations. As a result of the correlation extreme search, a set of actual coordinates $\mathbf{Q}_{i}, i=1,2, \ldots, K$ is formed.

The offset of the observable ellipse center in relation to the standard one (12) is calculated according to the coordinates $\mathbf{P}_{i}$ and $\mathbf{Q}_{i}, i=1,2, \ldots, K$ as an elevated sum of corrections.

$$
\Delta x=\sum_{i}\left(P_{i x}-Q_{i x}\right) P_{i x}^{2} / \sum_{i} P_{i x}^{2}, \quad \Delta y=\sum_{i}\left(P_{i y}-Q_{i y}\right) P_{i y}^{2} / \sum_{i} P_{i y}^{2} .
$$

Also it should be taken into account that accuracy of the correlation search is the greatest in the direction perpendicular to the counter boundary. Since plane (11) is perpendicular to the line connected the satellite and the Erath center, corrections to angles of the satellite orientation $\alpha$ and $\omega$ are found using simple geometric transformations:

$$
\Delta \alpha=\arctan \Delta x / \sqrt{X_{0}^{2}+Y_{0}^{2}+Z_{0}^{2}}, \Delta \omega=\arctan \Delta y / \sqrt{X_{0}^{2}+Y_{0}^{2}+Z_{0}^{2}} .
$$

These corrections are introduced into a matrix of setting angles $\mathbf{M}_{u}$ under the following geometric normalization according to (1)-(5).

Within flight tests of the satellite "Elektro-L", a lot of images have shown that the mentioned approach ensures accuracy of the geodetic connection of infrared range images about $2 \mathrm{~km}$ (under the spatial resolution of imagery $4 \mathrm{~km}$ ). Within the visible range the usage of counter points of the Earth is not reasonable because only a part of counter points is reflected within the most part of a day. In this case the geodetic connection is executed more effectively with approximate adjustment of the spacecraft orientation according to (17) with following adjustment of the geodetic connection using electronic maps [8].

\section{Conclusions}

The algorithms of normalization of information obtained from geostationary Earth remote sensing systems were developed. The algorithms have been experimentally tested within the flight tests of the geostationary satellite "Elektro-L". As a result high requirements to the quality of normalized images under significant distortions in original images were achieved to be fulfilled. Fulfilled estimations of normalization accuracy have shown that the geometrical normalization is carried out with accuracy not less than 1 pixel. 

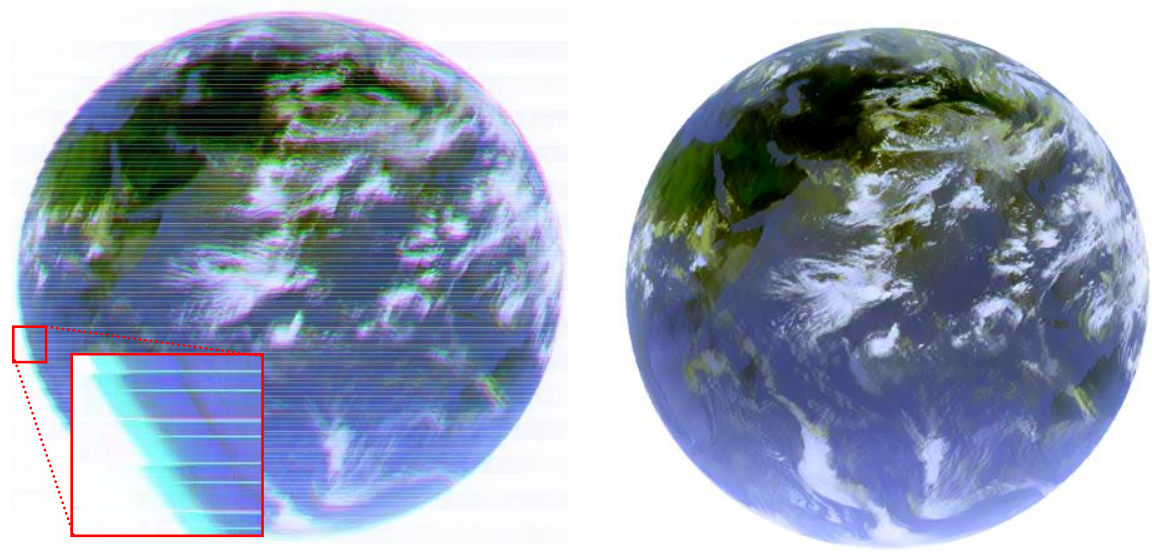

Figure 2. Images obtained from the satellite "Elektro-L" before (left) and after normalization (right).

Figure 2 shows the example of radiometric and geometric correction of the image obtained from the satellite "Elektro-L" in three infrared channels. On the left there is an original image where structural radiometric noise in the form of horizontal lines, color moirés because of geometric mismatches between spectral channels and image discontinuous structure are seen. On the right there is a result of normalization. Procession of data from one session of the Earth surface imagery by the satellite "Elektro-L" in 10 spectral channels with total volume of image information $1.5 \mathrm{~Gb}$ takes not more than 5 minutes using the server IBM x3850 having 4 dual core processors Intel Xeon $7120 \mathrm{~N} 4 x 3.0 \mathrm{GHz} / 667 \mathrm{MHz}$.

\section{References}

[1] "MSG End-User Requirements Document” EUMETSAT, EUM/MSG/SPE/013, 2004.

[2] Egoshkin N.A., Eremeev V.V, Kozlov E.P., Moskatinyev I.V., Moskvitin A.E. Geodetic binding of images from the geostationary satellite on a contour of a disk of the Earth and electronic maps [In Russian]. Current problems in remote sensing of the earth from space, 2009. Vol 6, P.132-138.

[3] Wolf R. LRIT/HRIT Global Specification. Coordination Group for Meteorological Satellites, 1999.

URL: http://www.eumetsat.int/website/wcm/idc/idcplg?IdcService=GET_FILE\&dDocName=PDF_C GMS_LRIT_HRIT_2_6\&RevisionSelectionMethod=LatestReleased\&Rendition=Web

[4] Bernd Jähne. Digital image processing. Springer, 2007.

[5] Zlobin V.K., Eremeev V.V. Aerospace image processing. Russian, Moskow, Fismathlit, 2006.

[6] Katamanov S.N. Automatic correction navigation method of FengYun-2 geosynchronous meteorological satellite imagery with pixel accuracy [In Russian]. Issledovanie Zemli iz Kosmosa, 2011. Vol. 2. c. 66-80.

[7] Katamanov S.N. Accurate HRIT image navigation for geostationary satellite MTSAT-1R. Current problems in remote sensing of the earth from space, 2012. Vol. 9. P. 97-105. 